\title{
EVOLUTION OF BERYLLIUM AND BORON IN THE INHOMOGENEOUS EARLY GALAXY
}

\author{
Takeru Ken Suzuki ${ }^{1,2}$, Yuzuru Yoshii ${ }^{3,4}$, and Toshitaka Kajino ${ }^{2,5,1}$
}

\begin{abstract}
A model of supernova-driven chemical evolution of the Galactic halo, recently proposed by Tsujimoto, Shigeyama, \& Yoshii (1999, ApJL, 519, 63), is extended in order to investigate the evolution of light elements such as $\mathrm{Be}$ and $\mathrm{B}(\mathrm{BeB})$, which are produced mainly through spallative reactions with Galactic cosmic rays (GCRs). In this model each supernova (SN) sweeps up the surrounding interstellar gas into a dense shell and directly enriches it with ejecta which consist of heavy elements produced in each Type II supernova with different progenitor masses. We propose a two-component source for GCRs such that both interstellar gas and fresh SN ejecta engulfed in the shell are accelerated by the shock wave. The released GCRs travel much faster than the expansion of the shell, and thus produce the BeB elements far outside the shell which will be incorporated in subsequent formation of shells arising from later SNe. As a result, stars formed from coeval shells are predicted to show a large scatter in the abundance of heavy elements, while exhibiting BeB abundances similar to that in the gas, with no appreciable scatter. This indicates that, contrary to heavy elements, stellar BeB abundances might be used as a good age indicator in the inhomogeneous Galactic halo.

The production of BeB at early epochs is dominated by the primary process through spallation of heavy GCRs, though being a minor component in the bulk of the GCR composition at present. We have calculated the frequency distribution of long-lived stars in the $\log (\mathrm{BeB} / \mathrm{H})-[\mathrm{Fe} / \mathrm{H}]$ plane and find that the contour of constant frequency covering a range of $-3<[\mathrm{Fe} / \mathrm{H}]<-1$ in this plane is consistent with the observed linear trend between BeB and Fe. We show from our calculations that there is an intriguing possibility of distinguishing between standard and non-standard Big-Bang nucleosynthesis models if BeB abundances in several hundred halo stars are observed in the future.
\end{abstract}

Subject headings: Galaxy: evolution — Galaxy: halo — nuclear reactions, cosmic rays stars: abundances — stars: Population II — supernovae: general — supernova remnants

\footnotetext{
${ }^{1}$ Department of Astronomy, School of Science, University of Tokyo, Bunkyo-ku, Tokyo, 113-0033 Japan

${ }^{2}$ Theoretical Astrophysics Division, National Astronomical Observatory, Mitaka, Tokyo, 181-8588 Japan; stakeru@yso.mtk.nao.ac.jp, kajino@th.nao.ac.jp

${ }^{3}$ Institute of Astronomy, School of Science, University of Tokyo, Mitaka, Tokyo, 181-8588 Japan; yoshii@ioa.s.u-tokyo.ac.jp

${ }^{4}$ Research Center for the Early Universe, School of Science, University of Tokyo,

Bunkyo-ku, Tokyo, 113-0033 Japan

${ }^{5}$ Department of Astronomical Science, Graduate University for Advanced Studies, Mitaka, Tokyo, 181-8588 Japan
} 


\section{INTRODUCTION}

The study of elemental abundance patterns in very metal-poor halo stars has suggested that stars with $-4<[\mathrm{Fe} / \mathrm{H}]<-2.5$ were born from individual supernova remnants (SNR), reflecting a different nucleosynthesis yield for each Type II supernova (SN II) with different progenitor mass (Shigeyama \& Tsujimoto 1998, hereafter ST98; Ishimaru \& Wanajo 1999). This indicates that previous models of chemical evolution in which stars are assumed to form from well-mixed, uniform gas cannot apply to the Galactic halo; as a result a unique age versus metallicity relation is not expected to hold for stars with $[\mathrm{Fe} / \mathrm{H}]<$ -2.5. Tsujimoto, Shigeyama, \& Yoshii (1999, hereafter TSY) have recently presented a new model for chemical evolution in the early Galactic halo, explicitly taking into account the expected inhomogeneous nature of the gas, and showed that their model is useful in interpreting the stellar abundances of heavy elements originating from SNe II.

The light elements such as $\mathrm{Be}$ and $\mathrm{B}(\mathrm{BeB})$ in halo stars, which are the focus of this paper, have usually been considered to originate from energetic protons and $\alpha$-particles in Galactic cosmic rays (GCRs) colliding with heavy nuclei of $\mathrm{C}, \mathrm{N}$, and $\mathrm{O}(\mathrm{CNO})$ in the interstellar medium (ISM) (e.g., Walker et al. 1985). While this secondary process naturally predicts a quadratic dependence of $\mathrm{BeB}$ abundance on metallicity, newly obtained data of stellar BeB abundances have, to the contrary, established a linear dependence (Boesgaard et al. 1999 for Be; Duncan et al. 1997 and Primas et al. 1999 for B).

To account for this discrepancy, many authors have considered possible primary processes for producing the BeB elements (e.g., Olive et al. 1994; Cassé et al. 1995; Vangioni-Flam et al. 1996; Lingenfelter et al. 1998; Vangioni-Flam et al. 1998; Higdon et al. 1998). In particular, Duncan et al. (1992) suggested that GCR CNO collide with protons and $\alpha$-particles in the ISM to produce BeB, which leads to their linear dependence on metallicity (see also Yoshii, Kajino, \& Ryan 1997; Ramaty et al. 1997). From a reanalysis of the elemental abundances of present-day GCRs and an examination of the physics of the SNR shock wave acceleration process, Meyer \& Ellison (1999, hereafter ME99) and Ellison \& Meyer (1999) have recently argued that most GCRs were accelerated out of the ISM or circumstellar material instead of SN ejecta, although they suggested that, owing to the lack of
ISM CNO at early epochs, GCR CNO out of fresh $\mathrm{SN}$ ejecta might play a role in producing the $\mathrm{BeB}$ elements.

In this paper, by incorporating both primary and secondary processes of GCR spallation of heavy elements, we can, for the first time, construct a model for the evolution of light elements which is fully consistent with the inhomogeneous chemical evolution of the early Galactic halo. The novelty of the result based on this model is that the frequency distribution of metal-poor stars in the $\log (\mathrm{BeB} / \mathrm{H})-[\mathrm{Fe} / \mathrm{H}]$ plane is predictable, along with the expected intrinsic abundance scatter.

\section{FORMULATION}

\subsection{Inhomogeneous Chemical Evolution}

We assume that the star-forming process is confined in separate clouds, which make up the entire halo of the early galaxy, and that the GCRs arise from multiple SN explosions therein. In our model a certain fraction $x_{\mathrm{III}}$ of cloud mass has turned into Pop III stars at time $t=0$ and the massive stars among them explode as Pop III SNe to initiate the chemical evolution in a given cloud. All stars of subsequent generations are assumed to form from the SNR shells behind the radiative shock front, which is supported by recent observations of the abundance patterns in metal-poor stars (ST98). The mass fraction of each shell turning into stars is taken as a constant, denoted by $\epsilon$. Following TSY, provided $x_{\mathrm{III}}<1 \times 10^{-2}$, a value of $\epsilon=4.3 \times 10^{-3}$ is used in the star formation rate given by

$$
\dot{M}_{*}(t)=\int_{\max \left(m_{t}, m_{\mathrm{SN}, l}\right)}^{m_{u}} d m \epsilon M_{\mathrm{sh}}(m, t) \frac{\phi(m)}{m} \dot{M}_{*}(t-\tau(m)),
$$

where $\tau(m)$ denotes the lifetime of a star with mass $m$, and $m_{t}$ is the stellar mass for which $\tau(m)=t$. The initial mass function, $\phi(m)$, having a Salpeter slope index of -1.35 , is adopted with upper and lower mass limits of $m_{u}=50 M_{\odot}$ and $m_{l}=0.05 M_{\odot}$. A lower mass limit of stars that explode as $\mathrm{SNe}$ is taken to be $m_{\mathrm{SN}, l}=10 M_{\odot}$. The mass of the shell is given by $M_{\mathrm{sh}}(m, t)=M_{\mathrm{ej}}(m)+M_{\mathrm{sw}}(m, t)$, where $M_{\mathrm{ej}}(m)$ is the mass of the SN ejecta and $M_{\mathrm{sw}}(m, t)$ is the mass of the gas swept up by the SNR. Since $M_{\mathrm{sw}}(m, t)$ is insensitive to $m$ and $t$, we here adopt $M_{\mathrm{sw}}(m, t)=$ $6.5 \times 10^{4} M_{\odot}(\mathrm{ST} 98 ; \mathrm{TSY})$.

The mass of the gas, $M_{\mathrm{g}}(t)$, and the abundance of the $j$-th heavy element in the gas, $Z_{j, \mathrm{~g}}(t)$, change 
with time according to eqs.(2) and (3) in TSY, respectively. Because of the inhomogeneous mixing of chemical compositions in the dense shells and the ISM, the abundance of the $j$-th heavy element in stars born at time $t$ in an SNR shell depends on the mass $m$ of SN progenitor, and is essentially given by $Z_{j, *}(m, t)=\left[M_{Z_{j}}(m)+Z_{j, \mathrm{~g}}(t) M_{\mathrm{sw}}(m, t)\right] / M_{\mathrm{sh}}(m, t)$ (see TSY).

Since the light elements $(\mathrm{LiBeB})$ are easily destroyed by stellar nuclear reactions at temperatures above a few $10^{6} \mathrm{~K}$, the abundance of the $L$ element in the gas $Z_{L, \mathrm{~g}}(t)$ changes through destruction by stellar lock-up and production by GCR reactions according to:

$$
\begin{aligned}
& \frac{d\left(Z_{L, \mathrm{~g}} M_{\mathrm{g}}\right)}{d t}=-\int_{\max \left(m_{t}, m_{\mathrm{SN}, l}\right)}^{m_{u}} d m Z_{L, *}(m, t) \epsilon M_{\mathrm{sh}}(m, t) \frac{\phi(m)}{m} \\
& \times \dot{M}_{*}(t-\tau(m))+\sum_{i=p \alpha, j=\mathrm{CNO}}\left\{\left\langle\sigma_{i j}^{L} F_{i}\right\rangle Z_{j, \mathrm{~g}}(t)\left(A_{L} / A_{j}\right)\right. \\
&\left.+\left\langle\sigma_{j i}^{L} F_{j}\right\rangle X_{i}(t)\left(A_{L} / A_{i}\right)\right\} M_{\mathrm{g}}(t),
\end{aligned}
$$

where $A_{i}$ is the mass number of the $i$-th element and $X_{i}$ is the abundance of hydrogen or helium. We define $\left\langle\sigma_{i j}^{L} F_{i}\right\rangle \equiv \int_{E_{\mathrm{th}}}^{\infty} \sigma_{i j}^{L}(E) F_{i}(E, t) S_{L}(E) d E$, where $\sigma_{i j}^{L}(E)$ is the cross section for the process of GCR projectile $i$ impinging on the ISM target $j$ to produce the $L$ element. $S_{L}(E)$ gives the retention fraction of $L$ products that can survive to be thermalized into the ISM, and $F_{i}(E, t)$ is the time-dependent flux of GCR projectile $i$, which is modeled in $\$ 2.2$. The stellar $L$ abundance is equal to that in the shell and is given by

$$
\begin{gathered}
Z_{L, *}(m, t)=\left[Z_{L, \mathrm{~g}}(t) M_{\mathrm{sw}}(m, t)+\sum_{i=p \alpha, j=\mathrm{CNO}}\right. \\
\left\{\left\langle\sigma_{i j}^{L} F_{i}\right\rangle Z_{j, \mathrm{~g}}(t)\left(A_{L} / A_{j}\right)+\left\langle\sigma_{j i}^{L} F_{j}\right\rangle X_{i}(t)\left(A_{L} / A_{i}\right)\right\} \\
\left.\times M_{\mathrm{sh}}(m, t) \Delta T\right] / M_{\mathrm{sh}}(m, t)
\end{gathered}
$$

where $\Delta T=10^{6} \mathrm{yr}$ is a typical diffusion time of a SNR shell (Shigeyama 1999, private communication). The first term in the numerator represents the mass of $L$ element swept up in the shell and the second term represents that mass produced during $\Delta T$ by GCRs originating from all the $\mathrm{SNe}$ that explode at time $t$.

\subsection{A Model of Time-Dependent GCR Flux}

The energy spectrum of the flux of propagated GCR projectiles, denoted by $F_{i}(E, t)$ in units of particles $\mathrm{s}^{-1} \mathrm{~cm}^{-2}(\mathrm{MeV} \text { per nucleon })^{-1}$, is expresssed as $F_{i}(E, t)=\left[\omega_{i}(E)\right]^{-1} \int_{E_{\mathrm{th}}}^{\infty} q_{i}\left(E^{\prime}, t\right) \exp \left[-\left(R_{i}\left(E^{\prime}\right)-\right.\right.$
$\left.\left.R_{i}(E)\right) / \Lambda\right] d E^{\prime}$ (Meneguzzi et al. 1971; Prantzos et al. 1993), where $q_{i}(E, t)$ is the source spectrum in particles $\mathrm{s}^{-1} \mathrm{~g}^{-1}(\mathrm{MeV} \text { per nucleon })^{-1}, \Lambda$ is the effective escape length in $\mathrm{g} \mathrm{cm}^{-2}, \omega_{i}(E)$ is the ionization energy losses in $\mathrm{MeV}\left(\mathrm{g} \mathrm{cm}^{-2}\right)^{-1}$ through a hydrogenhelium plasma with $\mathrm{X}=0.75$ and $\mathrm{Y}=0.25$, and $R_{\mathrm{i}}(E)$ is the ionization loss length in $\mathrm{g} \mathrm{cm}^{-2}$ (Northcliffe \& Schilling 1970). The effective escape length is given by $\Lambda^{-1}=\Lambda_{\mathrm{e}}^{-1}+\Lambda_{\mathrm{n}}^{-1}$ where $\Lambda_{\mathrm{e}}$ is the loss length of energetic particles against escape from the Galactic halo and $\Lambda_{\mathrm{n}}$ is that against nuclear destruction and decay. $\Lambda_{e}$ is proportional to the scale height $H$ in a cylindrical collapse of the halo (Prantzos et al. 1993) and approximately changes with time as $\Lambda_{\mathrm{e}} \propto 1-(a t)^{2}$, where $a^{-1}$ is the timescale of the collapse and a value of $1 \mathrm{Gyr}$ is used below. Although $\Lambda_{e}$ would be huge in the past, the maximum value of $\Lambda$ is bounded by $\Lambda_{\mathrm{n}} \simeq 200 \mathrm{~g} \mathrm{~cm}^{-2}$ for protons and $20 \mathrm{~g} \mathrm{~cm}^{-2}$ for $\alpha$ particles (Malaney \& Butler 1993).

We propose a new scenario in which GCRs originate from a combination of injected SN ejecta (primary origin) and swept-up ISM (secondary origin) in the SNR shell, which are then accelerated through the scattering back and forth across the shock. Their source spectrum $q_{i}(E, t)$ is assumed to have the same form, with its intensity proportional to the ejection rate of energetic particles associated with $\mathrm{SNe}$, that is:

$$
\begin{gathered}
q_{i}(E, t) \propto\left(E+E_{0}\right)^{-2.6} \int_{\max \left(m_{t}, m_{S N, l}\right)}^{m_{u}} d m \\
\times\left\{M_{Z_{i}}(m)+Z_{i, \mathrm{~g}}(t) f_{c r} M_{\mathrm{sw}}(m, t)\right\} \frac{\phi(m)}{A_{i} m} \dot{M}_{*}(t-\tau(m)),
\end{gathered}
$$

where $E_{0}=931 \mathrm{MeV}, M_{Z_{i}}(m)$ is the mass of the $i$ th heavy element synthesized and ejected from a star with mass $m$, and $f_{c r}$ is the fractional mass of the gas in the shell swept up while the SN explosion is able to accelerate ISM particles. If the acceleration were effective during the Sedov phase of the adiabatic shell expansion, a typical value of $f_{c r}$ would be $0.01-0.1$ (Taylor 1950; Sedov 1959). The absolute value of $q_{i}$ is chosen so that the predicted Be abundance from $F_{i}$ should agree with the observed abundance at $[\mathrm{Fe} / \mathrm{H}]=$ -3 . The composition of $\alpha$ particles relative to protons in GCRs is assumed to remain equal to a solar value of $F_{\alpha} / F_{p}=0.135$ (Lund 1989), but the ratio $F_{\mathrm{CNO}} / F_{p}$ is calculated by using eq.(值). 


\section{RESULTS AND DISCUSSION}

We have calculated the evolution of light and heavy elements in an inhomogeneous Galactic halo using the formulae of the preceding section. The values of the free parameters chosen in our model are: $x_{\text {III }}=10^{-6}, \epsilon=4.3 \times 10^{-3}$, and $f_{c r}=0.01$. The initial abundances of heavy elements are set to zero, but those of $\mathrm{BeB}$ elements are taken as equal to the predicted primordial abundances of $\log (\mathrm{Be} / \mathrm{H})=-17.9$ and $\log (\mathrm{B} / \mathrm{H})=-16.9$, based on the standard Big Bang nucleosynthesis (SBBN) model by Thomas et al. (1994).

Our results are shown in the color-coded frequency distribution of long-lived stars with $m<1 M_{\odot}$ in the $\log (\mathrm{Be} / \mathrm{H})-[\mathrm{Fe} / \mathrm{H}]$ plane (Fig.1) and in the $\log (\mathrm{B} / \mathrm{H})-$ $[\mathrm{Fe} / \mathrm{H}]$ plane (Fig.2). Both sets of frequency distributions have been convolved with a typical error $\sigma=0.15$ dex in the abundance measurements in order to directly compare with the Keck data for Be (Boesgaard et al. 1999) and the HST data for B (Duncan et al. 1997; Primas et al. 1999).

We define a probability density of finding one halo star within a unit area of $\Delta[\mathrm{Fe} / \mathrm{H}]=0.1 \times \Delta \log (\mathrm{BeB} / \mathrm{H})$ $=0.1$, normalized to unity when integrated over the entire area. The five contour lines shown in Figs.1 and 2 are, from the inside out, of constant probability density $10^{-2}, 10^{-3}, 10^{-4}, 10^{-6}$, and $10^{-8}$; the total probability integrated over the area inside each contour is $0.57,0.95,0.991,0.9999$, and 0.9999997 , respectively. Inspection of these figures shows that the area of 0.95 probability is in reasonable agreement with the distribution of observed data for both $\mathrm{Be}$ and $\mathrm{B}$, and that, except for the presumed existence of metal-free Pop III stars of the first generation, there should exist no stars below $[\mathrm{Fe} / \mathrm{H}] \approx-5$ because the SNR shells at each star formation site are enriched by the heavy-element ejecta from Pop III SNe. We note, however, that this value on the lower bound in the stellar metallicity is subject to the nucleosynthesis calculations adopted for SNe II.

The pattern of predicted abundance scatter in the $\log (\mathrm{BeB} / \mathrm{H})-[\mathrm{Fe} / \mathrm{H}]$ plane changes distinctly from lower to higher metallicity regions. This transition occurs at $[\mathrm{Fe} / \mathrm{H}] \sim-2.5$, below which the heavy-element mass of SN ejecta exceeds the heavy-element mass in the gas swept up in the SNR shell (ST98). Therefore, in the lower metallicity region $([\mathrm{Fe} / \mathrm{H}]<-2.5)$, stars formed at the same epoch from different SNR shells will exhibit a range of metallicities, reflecting a differ-

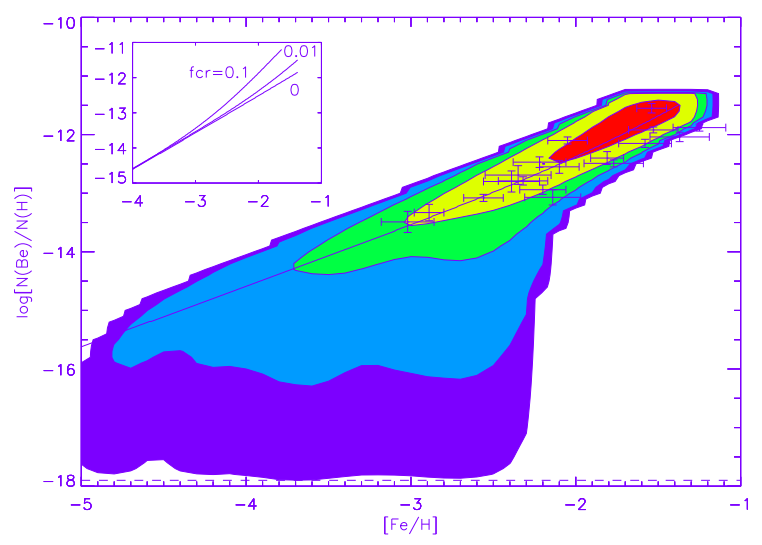

Fig. 1.- Color-coded frequency distribution of longlived stars in the $\log (\mathrm{Be} / \mathrm{H})-[\mathrm{Fe} / \mathrm{H}]$ plane, convolved with a Gaussian having $\sigma=0.15$ dex for both $\log (\mathrm{Be} / \mathrm{H})$ and $[\mathrm{Fe} / \mathrm{H}]$. The primorial Be abundance is taken from the SBBN model, and is indicated by the dashed line. The five contour lines, from the inside to the outside, correspond to those of constant probability density $10^{-2}, 10^{-3}, 10^{-4}, 10^{-6}$, and $10^{-8}$ in unit area of $\Delta[\mathrm{Fe} / \mathrm{H}]=0.1 \times \Delta \log (\mathrm{Be} / \mathrm{H})=0.1$. The thick line shows the $\log (\mathrm{Be} / \mathrm{H})-[\mathrm{Fe} / \mathrm{H}]$ relation for the gas for our standard choice of $f_{c r}=0.01$; the lines in the inset show those for various values of $f_{c r}$. The crosses represent the data with observational errors taken from Boesgaard et al. (1999).

ent nucleosynthesis yield for each SN II with different progenitor mass. On the other hand, the GCRs, which are accelerated either directly from each SN II or from swept-up ISM, can escape from the SNR shell at high speed without producing BeB through spallative reactions in the shell. As a result, most of $\mathrm{BeB}$ are produced in the ambient ISM far outside the shell, although the GCRs coming from outside impinge on targets in the shell to produce a small amount of $\mathrm{BeB}$ there. This accounts for the prediction that stars with $[\mathrm{Fe} / \mathrm{H}]<-2.5$ show a large scatter in the heavyelement abundance (along the horizontal axis), while exhibiting only a small scatter in the BeB abundance (along the vertical axis). It is therefore important to note that purely GCR-induced products like BeB can still be used as an good age indicator at early epochs, although the so-called age versus metallicity relation is broken.

The predicted scatter in $[\mathrm{Fe} / \mathrm{H}]$ becomes smaller towards higher $[\mathrm{Fe} / \mathrm{H}]$, so that the frequency distribu- 


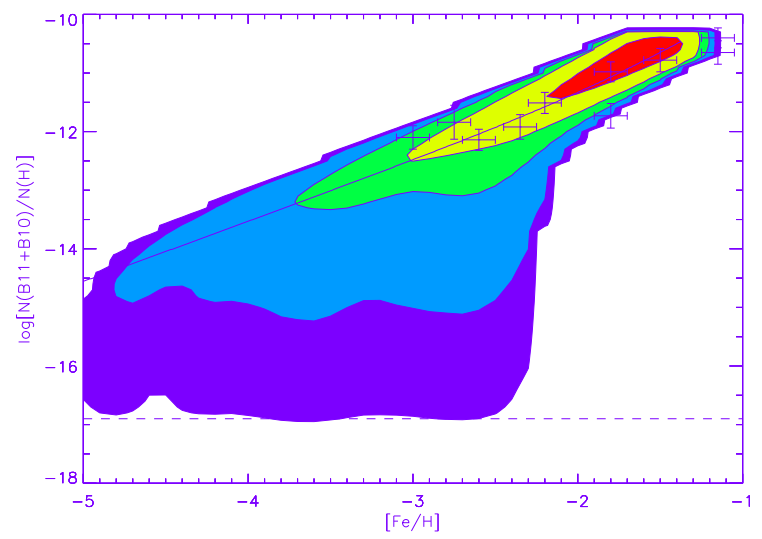

Fig. 2.- Color-coded frequency distribution of longlived stars in the $\log (\mathrm{B} / \mathrm{H})-[\mathrm{Fe} / \mathrm{H}]$ plane, as in Fig.1 but for $\mathrm{B}\left({ }^{10} \mathrm{~B}+{ }^{11} \mathrm{~B}\right)$. Crosses represent the data taken from Duncan et al. (1997) and Primas et al. (1999)

tion of stars in the $\log (\mathrm{BeB})-[\mathrm{Fe} / \mathrm{H}]$ plane converges to a linear relation $\mathrm{BeB} / \mathrm{H} \propto \mathrm{Fe} / \mathrm{H}$, as is observed over a range of $-3<[\mathrm{Fe} / \mathrm{H}]<-1$. This linear trend emerges when the primary GCR processes produce BeB. Notably, as suggested by ME99, the primary processes by GCR CNO impinging on ISM $p \alpha$, which are overwhelmed by the secondary processes at later epochs (including the present), become dominant at early epochs because of the lack of ISM CNO. This does not mean that most GCRs are of the primary origin. The mass ratio of primary to secondary GCRs in our model is $M_{\mathrm{ej}}(m) /\left(f_{c r} M_{\mathrm{sw}}(m, t)\right) \approx 10 /(0.01 \times 6.5 \times$ $\left.10^{4}\right) \sim 0.02$ (cf. eq.(化), which is not inconsistent with the recent results by ME99 that the GCRs originate mostly from the ISM. This ratio could also be argued by energetics between the kinetic energy released from SN and the input energy onto GCRs(Ramaty et al. 1997), which will be discussed in detail in our forthcoming paper.

As $[\mathrm{Fe} / \mathrm{H}]$ increases, the secondary GCR processes become dominant and a quadratic relation $\mathrm{BeB} / \mathrm{H} \propto$ $(\mathrm{Fe} / \mathrm{H})^{2}$ results. This changeover from a predicted linear to quadratic trend occurs at lower metallicity for larger $f_{c r}$, as shown in the inset of Fig.1. If a larger $f_{c r}$ is used, in a typical range of $0.01-0.1$ (Taylor 1950; Sedov 1959), the predicted relation deviates upwards from the observed linear trend. This deviation is partly offset by the effect of gas outflow from the cloud, which is known to flatten the slope of the $\log (\mathrm{BeB} / \mathrm{H})-[\mathrm{Fe} / \mathrm{H}]$ relation in the late phase of chemical evolution (e.g., Yoshii et al. 1997). In this paper we have taken into account this outflow only approximately by using a lower value of $f_{c r}=0.01$ as our standard. More BeB data for stars in the intermediate metallicity region $-2<[\mathrm{Fe} / \mathrm{H}]<-1$ are highly desirable to constrain $f_{c r}$, that is, the acceleration mechanism of GCRs as well as the rate of gas outflow from the cloud.

So far we have used the standard BBN model to set our assumed initial abundances of BeB elements, although their abundances are strongly dependent on the BBN model which is chosen. For example, if the density distribution in the early universe is not uniform as in the inhomogeneous BBN (IBBN) model, more $\mathrm{LiBeB}$ are synthesized in neutron-rich regions (Kajino \& Boyd 1990). In order to see how a nonstandard BBN model changes the result, we calculate the frequency distribution of long-lived stars in the $\log (\mathrm{Be} / \mathrm{H})-[\mathrm{Fe} / \mathrm{H}]$ plane using an optimal initial abundance of $\log (\mathrm{Be} / \mathrm{H})=-14.3$ taken from the IBBN model (Orito et al. 1997). The resulting distribution for the IBBN model is compared with the SBBN model in Fig.3, where the contour lines of constant probability density $10^{-4}$ and $10^{-5}$ in unit area of $\Delta[\mathrm{Fe} / \mathrm{H}]=0.1 \times \Delta \log (\mathrm{Be} / \mathrm{H})=0.1$ are shown. Since the total probability integrated over the area between the inner and outer contours is less than 0.01 , we need to measure $\mathrm{BeB}$ abundances in several hundred halo stars in order to distinguish the IBBN from the SBBN on a statistical basis. A more direct way to distiguish these alternative hypotheses is to identify true Pop III stars, the atmospheres of which consists of primeval gas. According to TSY, at least one halo star out of $10^{3-4}$ could be such a metal-free Pop III star. Such a large sample already exists (e.g., Beers 1999) and the discovery of Pop III stars may not be an unrealistic wish in the near future.

\section{SUMMARY}

We have presented a general approach for studying the GCR-induced evolution of the $L$ elements ( $\mathrm{LiBeB}$ ) in a manner consistent with an inhomogeneous chemical evolution of the Galactic halo. By adoption of a two-component source of GCRs, our model results include: (1) a prediction of the intrinsic scatter in $\mathrm{BeB}$ and $[\mathrm{Fe} / \mathrm{H}]$ abundances within the model, (2) a successful prediction of the observed linear trend between $\mathrm{BeB}$ and $\mathrm{Fe}$, (3) a proposal for using $\mathrm{BeB}$ as 


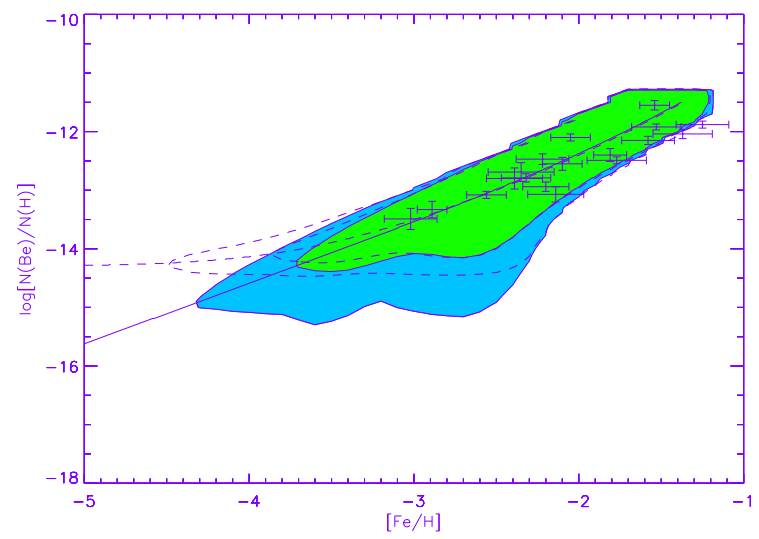

Fig. 3.- Frequency distribution of long-lived stars in the $\log (\mathrm{Be} / \mathrm{H})-[\mathrm{Fe} / \mathrm{H}]$ plane for two different primordial Be abundances predicted in the SBBN and IBBN models. The contour lines, from the inside to the outside, correspond to those of constant probability density $10^{-4}$ and $10^{-5}$ in unit area of $\Delta[\mathrm{Fe} / \mathrm{H}]=0.1 \times \Delta \log (\mathrm{Be} / \mathrm{H})=0.1$ for the SBBN (outer boundaries of two regions painted by different colors) and for the IBBN (dashed lines). The $\log (\mathrm{Be} / \mathrm{H})$ $[\mathrm{Fe} / \mathrm{H}]$ relation for the gas is shown by the thick line for the SBBN and by the dashed line for the IBBN. The crosses represent the data with observational errors taken from Boesgaard et al. (1999).

a cosmic clock, as an alternative to $[\mathrm{Fe} / \mathrm{H}]$, and (4) a method for possibly constraining the BBN model from future observations of metal-poor stars.

This work has been supported in part by the Grant-in-Aid for Scientific Research (10640236, 10044103) and the COE research (07CE2002) of the Ministry of Education, Science, Sports and Culture of Japan. We would like to thank T. C. Beers, T. Tsujimoto, and T. Shigeyama for many fruitful discussions.

\section{REFERENCES}

Beers, T. C. 1999, in ASP Conf. Ser. 165, The Third Stromlo Symp: The Galactic Halo, ed. B. K. Gibson, T. S. Axelrod, \& M. E. Putman (San Francisco: ASP), 206

Boesgaard, A. M., Deliyannis, C. P., King, J. R., Ryan, S. G., Vogt, S. S., \& Beers, T. C. 1999, AJ, 117,1549

Cassé, M., Lehoucq, R., \& Vangioni-Flam, E. 1995, Nature, 373, 318

Duncan, D. K., Lambert, D. L., \& Lemke, M. 1992, ApJ, 401, 584

Duncan, D. K., Primas, F., Rebull, L. M., Boesgaard, A. M., Deliyannis, C. P., Hobbs, L. M., King, J. R., \& Ryan, S. G. 1997, ApJ, 488, 338

Ellison, D. C., \& Meyer, J.-P. 1999 ASP Conf. Sers. in press (astro-ph 9905038)

Higdon, J. C., Lingenfelter, R. E., \& Ramaty, R. 1998 ApJ, 509, L33

Ishimaru, Y., \& Wanajo, S. 1999, ApJ, 511, L33

Kajino, T., \& Boyd, R. N. 1990, ApJ, 359, 267

Lingenfelter, R., E., Ramaty, R., \& Kozlovsky, B., 1998, 500, L53

Lund, N. 1989, in AIP Conf. Proc. 183, Cosmic, Abundances of Matter, ed. C. J. Waddington (New York: AIP), 111

Malaney, R. A., \& Butler, M. N. 1993, ApJ, 407, L73

Meneguzzi, M., Audouze, J., \& Reeves, H. 1971, A\&A, 15, 337

Meyer, J.-P., \& Ellison, D. C. 1999 ASP Conf. Sers. in press (astro-ph 9905037) (ME99)

Northcliffe, L. C., \& Schilling, R. F. 1970, Nuclear data Tables, A7, 233

Olive, K. A., Prantzos, N., Scully, S., \& VangioniFlam, E. 1994, ApJ, 424, 666

Orito, M., Kajino, T., Boyd, R. N., \& Mathews, G. J. 1997, ApJ, 488, 515

Prantzos, N., Cassé, M., \& Vangioni-Flam, E. 1993, ApJ, 403, 630 
Primas, F., Duncan, D. K., Peterson, R. C., \& Thorburn, J. A. 1999, A\&A, 343, 545

Ramaty, R., Kozlovsky, B., Lingenfelter, R. E., \& Reeves, H. 1997, ApJ, 488, 730

Sedov, L. I. 1959, Similarity and Dimensional Methods for Initial-Value Problem (New York: Wileyinterscience)

Shigeyama, T., \& Tsujimoto, T. 1998, ApJ, 507, L135 (ST98)

Taylor, G. I. 1950, Proc. R. Soc. London A, 201, 159

Thomas, D., Schramm, D. N., Olive, K. A., Mathews, G. J., Meyer, B. S., \& Fields, B. D. 1994, ApJ, 430, 291

Tsujimoto, T., Shigeyama, T., \& Yoshii, Y. 1999, ApJ, 519, L63 (TSY)

Vangioni-Flam, E., Cassé, M., Fields, B. D., \& Olive, K. A. 1996, ApJ, 468, 199

Vangioni-Flam, E., Ramaty, R., Olive, K. A., \& Cassé, M. 1998, A\&A, 337, 714

Walker, T. P., Mathews, G. J., \& Viola, V. E. 1985, ApJ, 299, 745

Yoshii, Y., Kajino, T., \& Ryan, S. G. 1997, ApJ, 485, 605

This 2-column preprint was prepared with the AAS LATEX macros $\mathrm{v} 4.0$. 